\title{
Why Governments Should Invest More in Fundamental Research
}

\begin{abstract}
Venki Ramakrishnan, ${ }^{\mathrm{a}, \mathrm{b}}$ Mejd Alsaric*
Venkatraman 'Venki' Ramakrishnan is the President of The Royal Society and Group Leader at the MRC Laboratory of Molecular Biology. In 2009 he shared the Nobel Prize in Chemistry 'for studies of the structure and function of the ribosome. In this interview he explains why governments should invest more in basic scientific research rather than simply on applied science and engineering. He also discusses interdisciplinarity, collaborations, and public engagement. See full interview at: https://youtu.be/GZPOpXPFWLE.
\end{abstract}

\section{Interdisciplinarity and Collaborations}

Mejd Alsari (MA). Can you explain how interdisciplinary research has contributed to the structural resolution of the ribosome?

Venki Ramakrishnan (VR). The first thing I could say is that at the Nobel Prizes in 2009 the Physics Prize was awarded for the development of CCD detectors and for the development of fibre optics, which allows large amounts of data to be moved.[1] The interesting thing is that the X-ray detectors that were used to solve the structure of the ribosome[2][3][4][5] at the time were based on CCD detectors. You could see right away how developments in physics had helped solve an important problem in biology using a sort of physical method. In that sense interdisciplinary work is extremely important, in that discoveries made in one field will often be used to drive other fields.

My own field, which is structural biology, is intrinsically interdisciplinary because it involves using physical techniques to attack biological problems. Sometimes what people mean by interdisciplinary is when people from different disciplines come together to attack a problem that neither of them could by themselves. That can often happen spontaneously. People who require analysis of data might go to a mathematician or a computer scientist and say "Look, I have this large-scale data and I don't know how to extract the information from it", and then they might collaborate. Or if you want to do a particular sort of experiment but you need some novel technique to attack it, you might go and talk to a physicist or a physical chemist and they might say "Actually if you were to use this technique it might help you obtain the kind of information you're looking for".

I think one has to be open-minded and encourage interdisciplinary work where it is needed. That means lowering barriers to interdisciplinary work. But I am not one of these missionaries who thinks that interdisciplinary work is always the answer or it should be promoted over everything else. That is just not the way science works. Some science is not interdisciplinary, is absolutely first-rate, and is making great advances. Other science, by definition, is interdisciplinary. Science should not be wedded to particular models, rather its goal is to not inhibit interactions. So if there is a barrier between talking to physicists, chemists, engineers, and so on, that is not a good thing. But if you remove these barriers then things will happen spontaneously as needed.

MA. Speaking of collaboration can you talk about the importance of international cooperation in research?

VR. I think for many things collaboration is essential. We are now collaborating with two groups. We have been collaborating

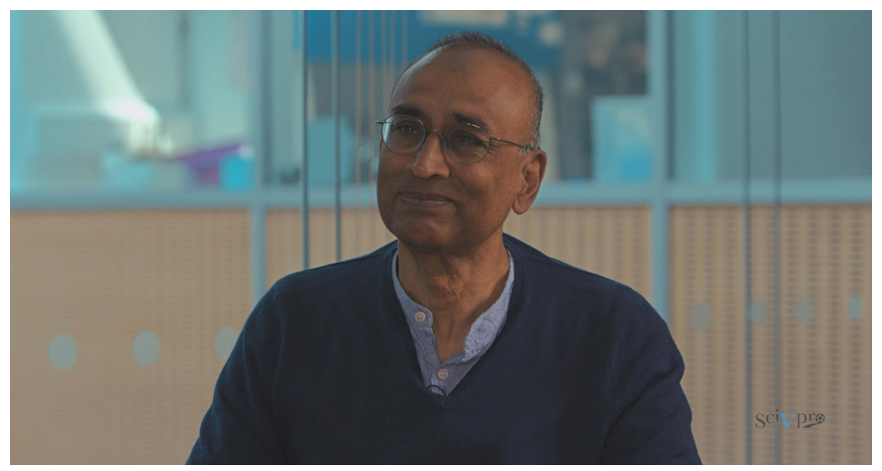

Figure 1 | "Future transformations in the economy are going to come out of the basic science of today and so a fraction of the research budget must always be set aside for very fundamental research", Venki Ramakrishnan.

with one group at the NIH on initiation of translation in yeast for almost 20 years now.[6] We have begun a collaboration with how this process works in human ribosomes with a group in California. These groups provide complementary expertise. We are focused on solving structures, but they have a lot of biochemical expertise and can also do genetics. There is quite a lot of natural collaboration.

There are also other types of collaborations such as the ones that involve very large-scale work like the Human Genome Project,[7] which was a very large-scale collaboration. The discovery of the Higgs boson involved scientists from many different countries working at CERN to attack a rather difficult problem.[8][9] The discovery of gravitational waves involved scientists from lots of different countries and in fact even equipment was based in lots of different countries to obtain a result.[10]

Science can vary from very small-scale independent efforts, which require no collaboration but simply require one or a few scientists to be really quite original and do their thing, to others, which require large-scale collaboration. There is not a one-size-fits-all model for science and I think we should not try to impose collaboration. That is not the way science works. In some cases you will need it. In other cases individuals working by themselves are going to make major contributions.

\section{Role of Fundamental Research in Emerging Markets}

MA. Could you talk about the role of governments on long-term investment in basic research? 
VR. I think governments invest in research because they know that it is good for the economy. Most Western countries prosper not because of cheap labour or raw materials, but because of knowledge. If you look at the growth of Europe, for most of history China and India were among the top economic countries in the world, and suddenly you see in the 1700s and 1800s Europe emerging as a big powerhouse and becoming the world's largest economy, and then was taken over by the US around the early 1900s.[11]

All of this has to do with the idea that knowledge is important for economic growth, it is what drives economic growth. Governments all realize that, but governments are under pressure. They want to spend money but they also want to know what you can get out of it. What governments need to realize is that virtually everything that you see today as part of driving the economy, major parts of the economy, they all came out of some basic curiosity-based research. If you look at something as simple as electricity, Michael Faraday, when he was discovering the laws of induction,[12] did not have the idea that it is going to become multitrillion-dollar industry worldwide. There is the same with batteries, video cameras, all of the inventions that go into a digital camera, the computers, the CCD chips. All of these things came out of basic science. It is the same with medicine. A fundamental understanding of physiology and cell biology and genetics has led to advances in medicine.

What I would argue is that it is very important to take basic research and make sure that it is used for applications and that applications are developed and commercialized in a way that benefits everybody. But it is also important to realize that the future transformations in the economy are going to come out of the basic science of today and so we have to realize that a fraction of the research budget must always be set aside for very fundamental research. Because we have no idea what it will lead to. For example the idea that high-energy physicists at CERN, because they wanted to share data, invented a protocol that now results in the World Wide Web, was not predictable.[13] This happened because of investment in fundamental science.

VR. I think governments need to always keep that in mind. Just as a farmer always has to set aside seed corn for next year's harvest, you do not want to use it all up.

MA. How can we convince then governments in some developing countries, including resource-rich countries, to invest in fundamental research?

I think we, in Britain, are doing a fairly good job and I think in the USA there has always been a recognition. For example the $\mathrm{Na}$ tional Institutes of Health, which is really for medical research, yet invests a huge amount of money in basic science.[14] I think there is a recognition. I would not say that there is not, but I think that there is a danger. There are other countries, which do not see the value of basic science and I think they are mistaken. They will never become first-rate economies unless they invest in a healthy mix of basic and applied science. Countries like Singapore, South Korea, and China,

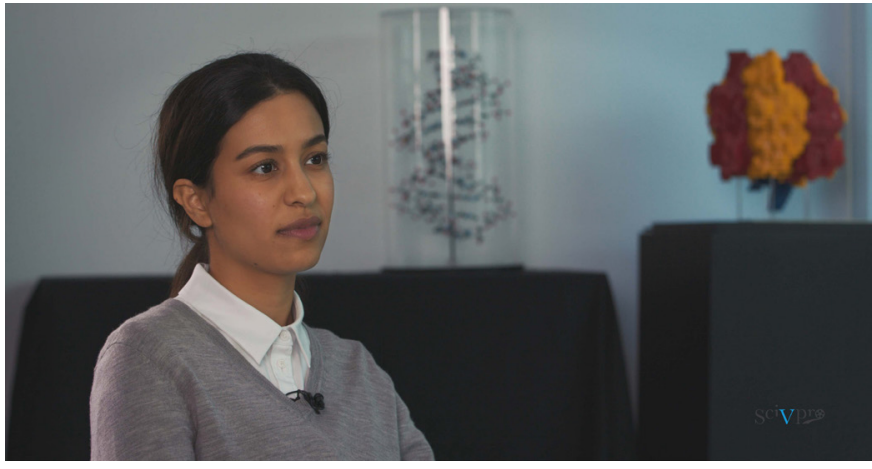

Figure 2 | Mejd Alsari, Visiting Research Associate at the Cavendish Laboratory (University of Cambridge).

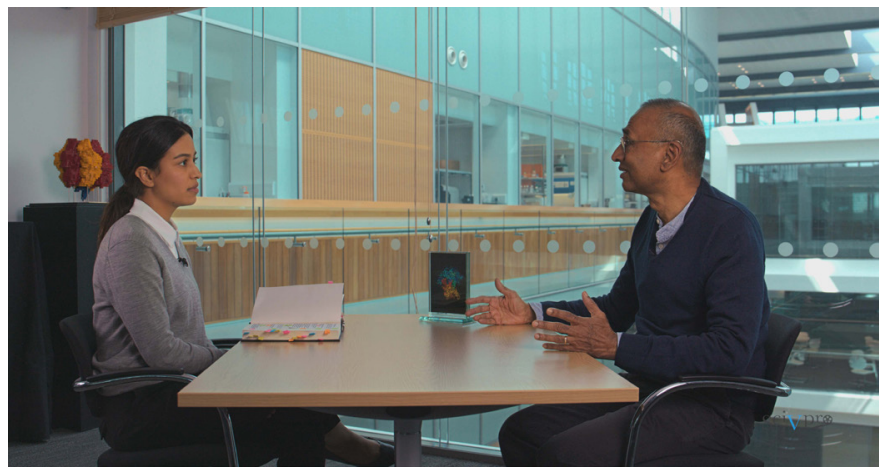

Figure 3 | Venki Ramakrishnan (right) and Mejd Alsari (left) at the MRC Laboratory of Molecular Biology.

they have all invested in basic research, also starting from nowhere. They also started from very little. There was very little research going on in all three of these countries and now they are all big powerhouses. I think it requires enlightened leadership at the top and it requires them to then hire good scientists, first-rate scientists maybe even from the West or returning from the West. But then they have got to give them independence and they have to trust them. That is sometimes hard for some countries.

\section{Public Engagement in Science}

MA. Public engagement in science. Why is that important?

VR. It is important for two reasons. The fundamental reason is that science is part of our culture. It is part of culture of humanity that we know things. We know for example how we actually inherit traits at the molecular level. That was amazing. For centuries no one understood why children were more similar to their parents than to other people and so on, why we did not give birth to animals instead of human beings. So nobody understood that and now we understand these things at a molecular level. We understand the universe might have begun with the Big Bang and so on. We understand how evolution works, we understand the nature of molecules. People until the $19^{\text {th }}$ century did not even know about molecules. These are all amazing triumphs of human understanding. If you went back 200 years and told people "We understand how the universe began and the molecular basis of heredity and how physiology works", and so on, they would think we were magicians. I think that is one reason to convey the excitement of science and the knowledge, which is really part of all of humanity.

The second reason is that in nearly all countries science is supported by the public through taxes. So it is really our duty as scientists to inform the public what is going on in science. How are we using your money to make discoveries and make advances, and occasionally improving life in various ways I think that is just part of our contract with the public.

\section{References}

[1] The Nobel Prize in Physics 2009, <https://www.nobelprize.org/prizes/physics/2009/summary/>, (2009).

[2] B. T. Wimberly, D. E. Brodersen, W. M. Clemons, R. J. Morgan-Warren, A. P. Carter, C. Vonrhein, T. Hartsch \& V. Ramakrishnan. Structure of the $30 \mathrm{~S}$ ribosomal subunit. Nature 407, 327-339, (2000).

[3] A. P. Carter, W. M. Clemons, D. E. Brodersen, R. J. Morgan-Warren, B. T. Wimberly \& V. Ramakrishnan. Functional insights from the structure of the 30 S ribosomal subunit and its interactions with antibiotics. Nature 407, 340, (2000).

[4] J. M. Ogle, D. E. Brodersen, W. M. Clemons, M. J. Tarry, A. P. Carter \& V. Ramakrishnan. Recognition of Cognate Transfer RNA by the 30 S Ribosomal Subunit. Science 292, 897, (2001).

[5] V. Ramakrishnan. Ribosome Structure and the Mechanism of Translation. Cell 108, 557-572, (2002). 
[6] National Institutes of Health, <https://www.nih.gov/>, (2019).

[7] The Human Genome Project, <https://www.genome.gov/human-genome-project>, (2019).

[8] S. Chatrchyan, V. Khachatryan, A. M. Sirunyan, A. Tumasyan, W. Adam, E. Aguilo, T. Bergauer, M. Dragicevic, J. Erö, C. Fabjan, M. Friedl, R. Frühwirth, V. M. Ghete, J. Hammer, M. Hoch, N. Hörmann, J. Hrubec, M. Jeitler, W. Kiesenhofer, V. Knünz et al. Observation of a new boson at a mass of $125 \mathrm{GeV}$ with the CMS experiment at the LHC. Physics Letters B 716, 30-61, (2012).

[9] G. Aad, T. Abajyan, B. Abbott, J. Abdallah, S. Abdel Khalek, A. A. Abdelalim, O. Abdinov, R. Aben, B. Abi, M. Abolins, O. S. AbouZeid, H. Abramowicz, H. Abreu, B. S. Acharya, L. Adamczyk, D. L. Adams, T. N. Addy, J. Adelman, S. Adomeit, P. Adragna et al. Observation of a new particle in the search for the Standard Model Higgs boson with the ATLAS detector at the LHC. Physics Letters B 716, 1-29, (2012).

[10] L. S. Collaboration, C. Virgo, B. P. Abbott, R. Abbott, T. D. Abbott, M. R. Abernathy, F. Acernese, K. Ackley, C. Adams, T. Adams, P. Addesso, R. X. Adhikari, V. B. Adya, C. Affeldt, M. Agathos, K. Agatsuma, N. Aggarwal, O. D. Aguiar, L. Aiello, A. Ain et al. Observation of Gravitational Waves from a Binary Black Hole Merger. Physical Review Letters 116, 061102, (2016).

[11] A. Maddison. Contours of the world economy 1-2030 AD: Essays in macro-economic history. (Oxford University Press, 2007).

[12] P. Day. The philosopher's tree: a selection of Michael Faraday's writings. (CRC Press, 1999).

[13] T. Berners-Lee, W. Hall, J. Hendler, N. Shadbolt \& D. J. Weitzner. Creating a Science of the Web. Science 313, 769, (2006).

[14] Impact of NIH Research, <https://www.nih.gov/about-nih/what-we-do/impact-nih-research>, (2019).

\section{Conflicts of Interest}

There are no conflicts to declare.

\section{Acknowledgements}

M.A. thanks Khalaf Al Habtoor, chairman of Al Habtoor Group, for sponsoring the video production. M.A. also thanks the MRC Laboratory of Molecular Biology and the Royal Society for arranging the interview with V.R.

\section{Author Contributions}

V.R. was interviewed by M.A., who wrote this manuscript.

\section{Keywords}

Public Funding, Fundamental Research, Emerging Markets, Interdisciplinarity, International Collaborations.

\section{Article Information}

This article is licensed by V. Ramakrishnan et al. under a Creative Commons Attribution 4.0 International License (CC BY 4.0). 